\title{
A Bernstein polynomial approach for solution of nonlinear integral equations
}

\author{
Neşe İşler Acara ${ }^{a}$ Ayşegül Daşcıoğlu ${ }^{b, *}$ \\ ${ }^{a}$ Department of Mathematics, Faculty of Arts and Sciences, Mehmet Akif Ersoy University, Burdur, Turkey. \\ ${ }^{b}$ Department of Mathematics, Faculty of Arts and Sciences, Pamukkale University, Denizli, Turkey. \\ Communicated by A. Atangana
}

\begin{abstract}
In this study, a collocation method based on the generalized Bernstein polynomials is derivated for solving nonlinear Fredholm-Volterra integral equations (FVIEs) in the most general form via the quasilinearization technique. Moreover, quadratic convergence and error estimate of the proposed method is analyzed. Some examples are also presented to show the accuracy and applicability of the method. (C)2017 All rights reserved.
\end{abstract}

Keywords: Bernstein polynomial approach, nonlinear integral equations, quasilinearization technique, collocation method. 2010 MSC: 45A05, 45G10, 65L60.

\section{Introduction}

Fredholm and Volterra integral equations are well-known that linear and nonlinear integral equations arise in many scientific fields such as the population dynamics, spread of epidemics and semi-conductor devices. The principal investigators of the theory of these equations are Vita Volterra (1860-1940) and Ivar Fredholm (1866-1927).

Quasilinearization pioneered by Bellman and Kalaba [6] is an effective technique that solves the nonlinear equations iteratively by a sequence of linear equations. Since this method is generalization of the Newton-Raphson method, it can be introduced by using the Taylor series expansion. The main advantage of this method is that it converges quadratically to the solution of the original equation. Some systematic studies of this property have been given in [1, 5-7, 11, 13-15, 18]. This method is also a powerful tool to obtain the approximate solution of nonlinear problems included such as differential equations $[1,3,5,9,14,17]$, functional differential equations $[2,7]$, integral equations $[13,15]$ and integro-differential equations [4, 18].

The Bernstein polynomials and their basis forms defined on the interval $[0,1]$ can be generalized to the interval $[a, b]$ by considering transformation $t=\frac{x-a}{b-a}$ as follows.

\footnotetext{
*Corresponding author

Email addresses: nisler@mehmetakif .edu.tr (Neşe İşler Acar), aakyuz@pau.edu.tr (Ayşegül Daşc1oğlu)
} 
Definition 1.1. The generalized Bernstein basis polynomials can be defined on $[a, b]$ by

$$
p_{i, n}(x)=\frac{1}{(b-a)^{n}}\left(\begin{array}{c}
n \\
i
\end{array}\right)(x-a)^{i}(b-x)^{n-i}, \quad i=0,1, \cdots, n .
$$

Definition 1.2. Let $y$ be a continuous function on the interval $[a, b]$. The generalized Bernstein polynomials of $n$-th-degree that linear combination of the generalized Bernstein basis polynomials $p_{i, n}(x)$ are defined by

$$
B_{n}(y ; x)=\sum_{i=0}^{n} y\left(a+\frac{(b-a) i}{n}\right) p_{i, n}(x) .
$$

Besides, the generalized Bernstein polynomials and their basis forms have some useful properties such as the positivity, continuity, recursion's relation, symmetry, unity partition of the basis set over the interval $[a, b]$, uniform approximation, differentiability and integrability. These properties can be shown easily by following the some studies given with the properties of the Bernstein polynomials and their basis forms $[8,10,12]$.

Theorem 1.3. If $y(x)$ is continuous function on the interval $[a, b]$, then $\lim _{n \rightarrow \infty} B_{n}(y ; x)=y(x)$ converges uniformly.

Proof. The above theorem is proved with the similar ways given for the proof of theorem presented by Phillips [16].

Definition 1.4. Nonlinear Fredholm-Volterra integral equation is defined as follows:

$$
g(x, y(x))=\lambda_{1} \int_{a}^{b} f(x, t, y(t)) d t+\lambda_{2} \int_{a}^{x} v(x, t, y(t)) d t, \quad x, t \in[a, b],
$$

where $\lambda_{1}$ and $\lambda_{2}$ are constants, $y(x)$ is an unknown function, $g:[a, b] \rightarrow \mathbb{R}$, the kernels $f:[a, b] \times[a, b] \rightarrow$ $\mathbb{R}$ and $v:[a, b] \times[a, b] \rightarrow \mathbb{R}$ are continuous functions satisfying a Lipschitz condition with respect to the last variables:

$$
\begin{array}{r}
g(x, z)-g(x, w) \leqslant L_{g}|z-w|, \\
|f(x, t, z)-f(x, t, w)| \leqslant L_{f}|z-w|, \\
|v(x, t, z)-v(x, t, w)| \leqslant L_{v}|z-w|,
\end{array}
$$

such that $\mathrm{L}_{\mathrm{g}}, \mathrm{L}_{\mathrm{f}}, \mathrm{L}_{v} \geqslant 0$ for $\mathrm{x}, \mathrm{t} \in[\mathrm{a}, \mathrm{b}]$ and $w, z \in \mathbb{R}$.

The reminder of this paper follows: In Section 2, a collocation method is developed iteratively to get the numerical solution of the nonlinear integral equations by means of the generalized Bernstein polynomials and quasilinearization technique. In Section 3, the uniqueness of the nonlinear FredholmVolterra integral equations is analyzed, and the error estimates of the proposed method are given. In Section 4, some nonlinear examples are considered for showing the applicability and efficiency of the method. Numerical results are also compared with different methods. Finally, some inferences of the study are mentioned in the last section.

\section{Method of solution}

In this paper, the purpose is to approximate the solution of nonlinear FVIE (1.1) by using the quasilinearization technique iteratively with the generalized Bernstein polynomials:

$$
y(x) \cong B_{n}(y ; x)=\sum_{i=0}^{n} y\left(a+\frac{(b-a) i}{n}\right) p_{i, n}(x) .
$$


Theorem 2.1. Let $\mathrm{x}_{\mathrm{s}} \in[\mathrm{a}, \mathrm{b}]$ be collocation point, $\mathrm{y} \in \mathbb{C}[\mathrm{a}, \mathrm{b}], \mathrm{g}$, $\mathrm{f}$ and $v$ have Taylor series expansion with respect to $y$. Then, nonlinear FVIE (1.1) has the following iteration matrix form:

$$
\left[\mathbf{G}_{\mathrm{r}} \mathbf{P}-\lambda_{1} \mathbf{F}_{\mathrm{r}}-\lambda_{2} \mathbf{V}_{\mathrm{r}}\right] \mathbf{Y}_{\mathrm{r}+1}=\mathbf{H}_{\mathrm{r}}, \quad \mathrm{r}=0,1, \cdots .
$$

Here matrices $\mathbf{G}_{\mathrm{r}}=\operatorname{diag}\left[g\left(\mathrm{x}_{\mathrm{s}}, \mathrm{y}_{\mathrm{r}}\left(\mathrm{x}_{\mathrm{s}}\right)\right)\right], \mathbf{P}=\left[p_{i, n}\left(\mathrm{x}_{\mathrm{s}}\right)\right], \mathbf{F}_{\mathrm{r}}=\left[F_{r, s, i}\right]$ and $\mathbf{V}_{\mathrm{r}}=\left[V_{\mathrm{r}, \mathrm{s}, \mathrm{i}}\right]$ are $(\mathrm{n}+1) \times(\mathrm{n}+1)$ matrices, $\mathbf{Y}_{\mathrm{r}+1}=\left[y_{\mathrm{r}+1}\left(\mathrm{a}+\frac{(\mathrm{b}-\mathrm{a}) \mathrm{i}}{\mathrm{n}}\right)\right]$ and $\mathbf{H}_{\mathrm{r}}=\left[h_{\mathrm{r}}\left(\mathrm{x}_{\mathrm{s}}\right)\right]$ are $(\mathrm{n}+1) \times 1$ matrices for $\mathrm{i}, \mathrm{s}=0,1, \cdots, \mathrm{n}$. Besides, elements of these matrices are defined as

$$
\begin{aligned}
h_{r}(x)= & g_{y}\left(x, y_{r}(x)\right) y_{r}(x)-g\left(x, y_{r}(x)\right)+\lambda_{1} \int_{a}^{b}\left[f\left(x, t, y_{r}(t)\right)-f_{y}\left(x, t, y_{r}(t)\right) y_{r}(t)\right] d t \\
& +\lambda_{2} \int_{a}^{x}\left[v\left(x, t, y_{r}(t)\right)-v_{y}\left(x, t, y_{r}(t)\right) y_{r}(t)\right] d t, \\
F_{r, s, i}= & \int_{a}^{b} f\left(x, t, y_{r}(t)\right) p_{i, n}(t) d t, \quad V_{r, s, i}=\int_{a}^{x} v\left(x, t, y_{r}(t)\right) p_{i, n}(t) d t .
\end{aligned}
$$

Proof. Since functions $g, f$ and $v$ are able to be expanded by Taylor series with respect to $y$, nonlinear FVIE can be expressed as a sequence of linear integral equations by using quasilinearization technique for $r=0,1, \cdots$ as follows

$$
\begin{aligned}
g_{y}\left(x, y_{r}(x)\right) y_{r+1}(x)= & g_{y}\left(x, y_{r}(x)\right) y_{r}(x)-g\left(x, y_{r}(x)\right) \\
& +\lambda_{1} \int_{a}^{b}\left[f\left(x, t, y_{r}(t)\right)+f_{y}\left(x, t, y_{r}(t)\right)\left(y_{r+1}(t)-y_{r}(t)\right)\right] d t \\
& +\lambda_{2} \int_{a}^{x}\left[v\left(x, t, y_{r}(t)\right)+v_{y}\left(x, t, y_{r}(t)\right)\left(y_{r+1}(t)-y_{r}(t)\right)\right] d t
\end{aligned}
$$

Here $g_{y}, f_{y}$ and $v_{y}$ are partial derivatives of $g, f$ and $v$ with respect to function $y, y_{0}(x)$ is an initial approximation function, $y_{r}(x)$ is always known function and $y_{r+1}(x)$ is obtained by the former one. By considering function $h_{r}(x)$ given in the expression of Theorem 2.1, the equation (2.3) can be rearranged shortly

$$
g_{y}\left(x, y_{r}(x)\right) y_{r+1}(x)=h_{r}(x)+\lambda_{1} \int_{a}^{b} f_{y}\left(x, t, y_{r}(t)\right) y_{r+1}(t) d t+\lambda_{2} \int_{a}^{x} v_{y}\left(x, t, y_{r}(t)\right) y_{r+1}(t) d t
$$

Since (1.1) has the generalized Bernstein polynomial solution and $y\left(x_{s}\right)=B_{n}\left(y ; x_{s}\right)(s=0,1, \cdots, n)$ from the collocation method, the expression (2.1) can be written as

$$
y_{r+1}\left(x_{s}\right)=\mathbf{P}\left(x_{s}\right) \mathbf{Y}_{r+1}, \quad r=0,1, \cdots .
$$

Substituting the collocation points and relation (2.5) into (2.4), we obtain the linear algebraic system

$$
g_{y}\left(x_{s}, y_{r}\left(x_{s}\right)\right) \mathbf{P}\left(x_{s}\right) \mathbf{Y}_{r+1}-\lambda_{1} \mathbf{F}_{r}\left(x_{s}\right) \mathbf{Y}_{r+1}-\lambda_{2} \mathbf{V}_{r}\left(x_{s}\right) \mathbf{Y}_{r+1}=h_{r}\left(x_{s}\right) .
$$

Here $\mathbf{F}_{\mathbf{r}}\left(\mathrm{x}_{\mathrm{s}}\right)$ and $\mathbf{V}_{\mathbf{r}}\left(\mathrm{x}_{\mathrm{s}}\right)$ are denoted by

$$
\mathbf{F}_{r}\left(x_{s}\right)=\int_{a}^{b} f_{y}\left(x, t, y_{r}(t)\right) \mathbf{P}(t) d t=\left[\begin{array}{llll}
F_{r, s, 0} & F_{r, s, 1} & \ldots & F_{r, s, n}
\end{array}\right]
$$




$$
\mathbf{V}_{r}\left(x_{s}\right)=\int_{a}^{x} v_{y}\left(x, t, y_{r}(t)\right) \mathbf{P}(t) d t=\left[\begin{array}{llll}
V_{r, s, 0} & V_{r, s, 1} & \ldots & V_{r, s, n}
\end{array}\right] .
$$

Considering the matrices

$$
\begin{aligned}
& \mathbf{G}_{r}=\left[\begin{array}{cccc}
g_{y}\left(x_{0}, y_{r}\left(x_{0}\right)\right) & 0 & \ldots & 0 \\
0 & g_{y}\left(x_{1}, y_{r}\left(x_{1}\right)\right) & \ldots & 0 \\
\vdots & \vdots & \ddots & \vdots \\
0 & 0 & \cdots & g_{y}\left(x_{n}, y_{r}\left(x_{n}\right)\right)
\end{array}\right], \\
& \mathbf{F}_{\mathbf{r}}=\left[\begin{array}{c}
\mathbf{F}_{\mathbf{r}}\left(x_{0}\right) \\
\mathbf{F}_{\mathbf{r}}\left(x_{1}\right) \\
\vdots \\
\mathbf{F}_{\mathbf{r}}\left(x_{\mathrm{n}}\right)
\end{array}\right], \quad \mathbf{V}_{\mathbf{r}}=\left[\begin{array}{c}
\mathbf{V}_{\mathbf{r}}\left(x_{0}\right) \\
\mathbf{V}_{\mathbf{r}}\left(x_{1}\right) \\
\vdots \\
\mathbf{V}_{\mathbf{r}}\left(x_{\mathfrak{n}}\right)
\end{array}\right], \quad \mathbf{H}_{\mathrm{r}}=\left[\begin{array}{c}
h_{\mathrm{r}}\left(x_{0}\right) \\
h_{\mathrm{r}}\left(x_{1}\right) \\
\vdots \\
h_{\mathrm{r}}\left(x_{\mathfrak{n}}\right)
\end{array}\right],
\end{aligned}
$$

for $s=0,1, \cdots, n$, the equation (2.6) can be written as matrix form (2.2). This completes the proof.

Let the following steps be given to solve the nonlinear FVIE (1.1).

Step 1. The equation (2.2) can also be written in the compact form

$$
\mathbf{W}_{\mathrm{r}} \mathbf{Y}_{\mathrm{r}+1}=\mathbf{H}_{\mathrm{r}} \quad \text { or } \quad\left[\mathbf{W}_{\mathrm{r}} ; \mathbf{H}_{\mathrm{r}}\right], \quad \mathbf{r}=0,1, \cdots,
$$

so that $\mathbf{W}_{\mathrm{r}}=\mathbf{G}_{\mathrm{r}} \mathbf{P}-\lambda_{1} \mathbf{F}_{\mathrm{r}}-\lambda_{2} \mathbf{V}_{\mathrm{r}}$. This equation corresponds to a linear algebric equation system with $y_{r+1}$ unknown coefficients for iterations $r$.

Step 2. An initial approximation function $y_{0}(x)$ should be selected for computing the $\mathbf{W}_{\mathrm{r}}$ and $\mathbf{H}_{\mathrm{r}}$. This function can be determined as source or constant function roughly.

Step 3. Since nonlinear integral equations reduce to a sequence of linear integral equations via the quasilinearization technique, we do not need to use any solution techniques of nonlinear integral equations. As linear equation system, if $\operatorname{rank}\left(\mathbf{W}_{\mathrm{r}}\right)=\operatorname{rank}\left(\mathbf{W}_{\mathrm{r}} ; \mathbf{H}_{\mathrm{r}}\right)=\mathrm{n}+1$, then solution of this system is uniquely determined. The system can also be solved by the Gauss elimination, generalized inverse, LU and QR factorization methods.

\section{Convergence and error analysis}

Definition 3.1. Error is denoted by $e_{n}(x)=y(x)-y_{r+1}(x)$ such that $y(x)$ is an exact solution and $y_{r+1}(x)=B_{n}\left(y_{r+1} ; x\right)$ is a generalized Bernstein approximate solution. Then absolute and mean errors can be numerically computed at the collocation points respectively by

$$
\left|e_{n}\left(x_{s}\right)\right|=\left|y\left(x_{s}\right)-y_{r+1}\left(x_{s}\right)\right|, \quad e_{\text {mean }}=\frac{1}{n+1} \sum_{s=0}^{n}\left|e_{n}\left(x_{s}\right)\right| .
$$

Definition 3.2. Let $E_{r}\left(x_{s}\right)=y_{r+1}\left(x_{s}\right)-y_{r}\left(x_{s}\right)$ be error on the collocation points $x_{s} \in[a, b]$ for the r-th iteration function. Then absolute and maximum errors can be expressed as follows:

$$
\left|E_{r}\left(x_{s}\right)\right|=\left|y_{r+1}\left(x_{s}\right)-y_{r}\left(x_{s}\right)\right|, \quad \text { and } \quad E_{\max }=\max _{x_{s} \in[a, b]}\left|E_{r}\left(x_{s}\right)\right| \text {. }
$$

Theorem 3.3 (Uniqueness Theorem). Let $\mathrm{g} \in \mathbb{C}[\mathrm{a}, \mathrm{b}]$ and $\mathrm{f}, v \in \mathbb{C}\left([\mathrm{a}, \mathrm{b}]^{2}\right)$ satisfy the Lipschitz condition with respect to the last variables and $\mathrm{T}: \mathbb{C}[\mathrm{a}, \mathrm{b}] \rightarrow \mathbb{C}[\mathrm{a}, \mathrm{b}]$ be a mapping where

$$
\operatorname{Ty}(x)=g(x, y(x))-\lambda_{1} \int_{a}^{b} f(x, t, y(t)) d t-\lambda_{2} \int_{a}^{x} v(x, t, y(t)) d t .
$$

Then (1.1) has a unique solution whenever $0<\alpha<1, \alpha=\mathrm{L}_{g}+(\mathrm{b}-\mathrm{a})\left[\left|\lambda_{1}\right| \mathrm{L}_{\mathrm{f}}+\left|\lambda_{2}\right| \mathrm{L}_{v}\right]$. 
Proof. Since $g, f$ and $v$ satisfy the Lipschitz condition with respect to the last variables $y$ and $y^{*}$, we have the following inequality for $y, y^{*} \in \mathbb{C}[a, b]$ :

$$
\begin{aligned}
\left|T y(x)-T y^{*}(x)\right| \leqslant & \left|g(x, y(x))-g\left(x, y^{*}(x)\right)\right|+\left|\lambda_{1}\right| \int_{a}^{b}\left|f(x, t, y(t))-f\left(x, t, y^{*}(t)\right)\right| d t \\
& +\left|\lambda_{2}\right| \int_{a}^{x}\left|v(x, t, y(t))-v\left(x, t, y^{*}(t)\right)\right| d t \\
\leqslant & L_{g}\left|y(x)-y^{*}(x)\right|+\left|\lambda_{1}\right| L_{f} \int_{a}^{b}\left|y(t)-y^{*}(t)\right| d t+\left|\lambda_{2}\right| L_{v} \int_{a}^{x}\left|y(t)-y^{*}(t)\right| d t .
\end{aligned}
$$

Considering the definition of maximum norm for one variable function, the inequality becomes

$$
\begin{aligned}
\left\|T y-T y^{*}\right\|_{\infty} & \leqslant L_{g}\left\|y-y^{*}\right\|_{\infty}+\left|\lambda_{1}\right| L_{f}\left\|y-y^{*}\right\|_{\infty} \int_{a}^{b} d t+\left|\lambda_{2}\right| L_{v}\left\|y-y^{*}\right\|_{\infty} \int_{a}^{x} d t \\
& \leqslant L_{g}\left\|y-y^{*}\right\|_{\infty}+\left|\lambda_{1}\right| L_{f}(b-a)\left\|y-y^{*}\right\|_{\infty}+\left|\lambda_{2}\right| L_{v}(b-a)\left\|y-y^{*}\right\|_{\infty} \\
& \leqslant\left[L_{g}+(b-a)\left(\left|\lambda_{1}\right| L_{f}+\left|\lambda_{2}\right| L_{v}\right)\right]\left\|y-y^{*}\right\|_{\infty}
\end{aligned}
$$

for all $x, t \in[a, b]$. Denoting $\alpha=L_{g}+(b-a)\left[\left|\lambda_{1}\right| L_{f}+\left|\lambda_{2}\right| L_{v}\right]$, the inequality is $\left\|T y-T y^{*}\right\| \leqslant \alpha\left\|y-y^{*}\right\|$. Under the condition $0<\alpha<1$, by Banach fixed-point theorem, (1.1) has a unique solution and this completes the proof.

Theorem 3.4. Suppose that $\mathrm{y}_{0}(\mathrm{x})$ and $\mathrm{y}_{\mathrm{r}}(\mathrm{x})$ are the first and $\mathrm{r}$-th iteration functions, $\mathrm{g} \in \mathbb{C}^{2}[\mathrm{a}, \mathrm{b}]$, $\mathrm{f}$ and $v \in \mathbb{C}^{2}\left([\mathrm{a}, \mathrm{b}]^{2}\right)$. Then the following quadratic convergence and error estimate hold $\left\|\mathrm{E}_{\mathrm{r}}\right\|_{\infty} \leqslant \sigma\left\|\mathrm{E}_{\mathrm{r}-1}\right\|_{\infty}^{2}$ and $\left\|\mathrm{E}_{\mathrm{r}}\right\|_{\infty} \leqslant \frac{\left(\sigma\left\|\mathrm{E}_{1}\right\|_{\infty}\right)^{2^{r}}}{\sigma}$ such that $\sigma=\frac{\mathrm{M}_{2}+(\mathrm{b}-\mathrm{a})\left[\left|\lambda_{1}\right| \mathrm{L}_{1}+\left|\lambda_{2}\right| \mathrm{L}_{2}\right]}{2\left[\mathrm{M}_{1}-(\mathrm{b}-\mathrm{a})\left(\left|\lambda_{1}\right| \mathrm{K}_{1}+\left|\lambda_{2}\right| \mathrm{K}_{2}\right)\right]}$ a positive constant. If the quantity $\left\|\mathrm{E}_{1}\right\|_{\infty}<1$, then $\lim _{\mathrm{r} \rightarrow \infty}\left\|\mathrm{E}_{\mathrm{r}}\right\|_{\infty}=0$.

Proof. Applying the quasilinearization technique to the following equality

$$
g\left(x, y_{r+1}\right)-g\left(x, y_{r}\right)=\lambda_{1} \int_{a}^{b}\left\{f\left(x, t, y_{r+1}\right)-f\left(x, t, y_{r}\right)\right\} d t+\lambda_{2} \int_{a}^{x}\left\{v\left(x, t, y_{r+1}\right)-v\left(x, t, y_{r}\right)\right\} d t
$$

we have

$$
\begin{aligned}
g_{y}\left(x, y_{r}\right)\left(y_{r+1}-y_{r}\right)= & -\left\{g\left(x, y_{r}\right)-g\left(x, y_{r}-1\right)-g_{y}\left(x, y_{r-1}\right)\left(y_{r}-y_{r}-1\right)\right\} \\
& +\lambda_{1} \int_{a}^{b}\left\{f\left(x, t, y_{r}\right)-f\left(x, t, y_{r-1}\right)-f_{y}\left(x, t, y_{r}-1\right)\left(y_{r}-y_{r-1}\right)\right\} d t \\
& +\lambda_{2} \int_{a}^{x}\left\{v\left(x, t, y_{r}\right)-v\left(x, t, y_{r}-1\right)-v_{y}\left(x, t, y_{r-1}\right)\left(y_{r}-y_{r-1}\right)\right\} d t \\
& +\lambda_{1} \int_{a}^{b} f_{y}\left(x, t, y_{r}\right)\left(y_{r+1}-y_{r}\right) d t++\lambda_{2} \int_{a}^{x} v_{y}\left(x, t, y_{r}\right)\left(y_{r+1}-y_{r}\right) d t
\end{aligned}
$$

Since $g \in \mathbb{C}^{2}[a, b]$, $f$ and $v \in \mathbb{C}^{2}\left([a, b]^{2}\right)$, the following equations can be written from mean value theorem 


$$
\begin{gathered}
g\left(x, y_{r}\right)-g\left(x, y_{r-1}\right)-g_{y}\left(x, y_{r-1}\right)\left(y_{r}-y_{r-1}\right)=\frac{1}{2}\left(y_{r}-y_{r-1}\right)^{2} g_{y y}(x, \alpha), \\
f\left(x, t, y_{r}\right)-f\left(x, t, y_{r-1}\right)-f_{y}\left(x, t, y_{r-1}\right)\left(y_{r}-y_{r-1}\right)=\frac{1}{2}\left(y_{r}-y_{r-1}\right)^{2} f_{y y}(x, t, \beta), \\
v\left(x, t, y_{r}\right)-v\left(x, t, y_{r-1}\right)-v_{y}\left(x, t, y_{r-1}\right)\left(y_{r}-y_{r-1}\right)=\frac{1}{2}\left(y_{r}-y_{r-1}\right)^{2} v_{y y}(x, t, \gamma),
\end{gathered}
$$

where $y_{r-1}<\alpha, \beta, \gamma<y_{r}$. Substituting these equations into (3.1), the equation becomes

$$
\begin{aligned}
g_{y}\left(x, y_{r}\right)\left(y_{r+1}-y_{r}\right)= & -\frac{1}{2}\left(y_{r}-y_{r-1}\right)^{2} g_{y y}(x, \alpha)+\lambda_{1} \int_{a}^{b} \frac{1}{2}\left(y_{r}-y_{r-1}\right)^{2} f_{y y}(x, t, \beta) d t \\
& +\lambda_{2} \int_{a}^{x} \frac{1}{2}\left(y_{r}-y_{r-1}\right)^{2} v_{y y}(x, t, \gamma) d t+\lambda_{1} \int_{a}^{b} f_{y}\left(x, t, y_{r}\right)\left(y_{r+1}-y_{r}\right) d t \\
& +\lambda_{2} \int_{a}^{x} v_{y}\left(x, t, y_{r}\right)\left(y_{r+1}-y_{r}\right) d t .
\end{aligned}
$$

By definition of the maximum norm for one and two variables functions, the following inequality is obtained

$$
\begin{aligned}
\left\|g_{y}\right\|_{\infty}\left\|y_{r+1}-y_{r}\right\|_{\infty} \leqslant & \frac{1}{2}\left\|y_{r}-y_{r-1}\right\|_{\infty}^{2}\left\|g_{y y}\right\|_{\infty}+\frac{1}{2}\left|\lambda_{1}\right|\left\|y_{r}-y_{r-1}\right\|_{\infty}^{2}\left\|f_{y y}\right\|_{\infty}(b-a) \\
& +\frac{1}{2}\left|\lambda_{2}\right|\left\|y_{r}-y_{r-1}\right\|_{\infty}^{2}\left\|v_{y y}\right\|_{\infty}(b-a)+\left|\lambda_{1}\right|\left\|f_{y}\right\|_{\infty}\left\|y_{r+1}-y_{r}\right\|_{\infty}(b-a) \\
& +\left|\lambda_{2}\right|\left\|v_{y}\right\|_{\infty}\left\|y_{r+1}-y_{r}\right\|_{\infty}(b-a)
\end{aligned}
$$

\section{Denoting}

$$
\begin{aligned}
\left\|g_{y}\right\|_{\infty} & =\max _{x \in[a, b]}\left|g_{y}\left(x, y_{r}(x)\right)\right|=M_{1}, \\
\left\|g_{y y}\right\|_{\infty} & =\max _{x \in[a, b]}\left|g_{y y}(x, \alpha(x))\right|=M_{2}, \\
\left\|f_{y}\right\|_{\infty} & =\max _{x, t \in[a, b]}\left|f_{y}\left(x, t, y_{r}\right)\right|=K_{1}, \\
\left\|v_{y}\right\|_{\infty} & =\max _{x, t \in[a, b]}\left|v_{y}\left(x, t, y_{r}\right)\right|=K_{2}, \\
\left\|f_{y y}\right\|_{\infty} & =\max _{x, t \in[a, b]}\left|f_{y y}(x, t, z)\right|=L_{1}, \\
\left\|v_{y y}\right\|_{\infty} & =\max _{x, t \in[a, b]}\left|v_{y y}(x, t,)\right|=L_{2},
\end{aligned}
$$

and

$$
\max _{x \in[a, b]}(x-a)=b-a,
$$

the above inequality becomes

$$
\begin{aligned}
M_{1}\left\|y_{r+1}-y_{r}\right\|_{\infty} \leqslant & \frac{M_{2}}{2}\left\|y_{r}-y_{r-1}\right\|_{\infty}^{2}+\frac{L_{1}}{2}\left|\lambda_{1}\right|(b-a)\left\|y_{r}-y_{r-1}\right\|_{\infty}^{2} \\
& +\frac{L_{2}}{2}\left|\lambda_{2}\right|(b-a)\left\|y_{r}-y_{r-1}\right\|_{\infty}^{2}+\left|\lambda_{1}\right| K_{1}(b-a)\left\|y_{r+1}-y_{r}\right\|_{\infty} \\
& +\left|\lambda_{2}\right| K_{2}(b-a)\left\|y_{r+1}-y_{r}\right\|_{\infty} .
\end{aligned}
$$

This inequality can be rearranged as

$$
\left\|E_{r}\right\|_{\infty} \leqslant\left\{\frac{M_{2}+\left|\lambda_{1}\right| L_{1}(b-a)+\left|\lambda_{2}\right| L_{2}(b-a)}{2\left[M_{1}-\left|\lambda_{1}\right| K_{1}(b-a)-\left|\lambda_{2}\right| K_{2}(b-a)\right]}\right\}\left\|E_{r-1}\right\|_{\infty}^{2},
$$




$$
\left\|\mathrm{E}_{\mathrm{r}}\right\|_{\infty} \leqslant \sigma\left\|\mathrm{E}_{\mathrm{r}-1}\right\|_{\infty}^{2}
$$

where $\sigma=\frac{M_{2}+\left|\lambda_{1}\right| L_{1}(b-a)+\left|\lambda_{2}\right| L_{2}(b-a)}{2\left[M_{1}-\left|\lambda_{1}\right| K_{1}(b-a)-\left|\lambda_{2}\right| K_{2}(b-a)\right]}$ a positive constant. This equation shows that convergence is quadratic if there is convergence at all. We rewrite this equation as

$$
\begin{aligned}
\left\|\mathrm{E}_{\mathrm{r}}\right\|_{\infty} & \leqslant \sigma\left\|\mathrm{E}_{\mathrm{r}-1}\right\|_{\infty}^{2}, \\
\left\|\mathrm{E}_{\mathrm{r}-1}\right\|_{\infty} & \leqslant \sigma\left\|\mathrm{E}_{\mathrm{r}-2}\right\|_{\infty}^{2}, \\
& \vdots \\
\left\|\mathrm{E}_{2}\right\|_{\infty} & \leqslant \sigma\left\|\mathrm{E}_{1}\right\|_{\infty}^{2} .
\end{aligned}
$$

By the use of substitutions, the new inequality is

$$
\left\|E_{r}\right\|_{\infty} \leqslant \sigma\left\|E_{r-1}\right\|_{\infty}^{2} \leqslant \sigma\left(\sigma\left\|E_{r-2}\right\|_{\infty}^{2}\right)^{2} \leqslant \ldots \leqslant \sigma\left[\sigma^{2^{r}-2}\left\|E_{r-3}\right\|_{\infty}^{2^{r}}\right]=\left[\sigma\left\|E_{1}\right\|_{\infty}\right]^{2^{r}} / \sigma .
$$

If the quantity $\sigma\left\|E_{1}\right\|_{\infty}<1$, then $\lim _{r \rightarrow \infty}\left\|E_{r}\right\|_{\infty}=0$. It completes the proof.

\section{Numerical results}

Two examples including nonlinear integral equations are considered for analyzing the applicability and accuracy of the proposed Bernstein collocation method. The numerical results obtained on the collocation points $a+\frac{(b-a) s}{n}, s=0,1, \cdots, n$ are presented and compared with the other methods.

Example 4.1. Consider the following nonlinear Volterra integral equation:

$$
y(x)=2-e^{x}+\int_{0}^{x} e^{x-t} y^{2}(t) d t, \quad 0 \leqslant x \leqslant 1
$$

Exact solution of the above equation is $y(x)=1$. Let $y_{0}(x)=2-e^{x}$ be initial approximation function.

In Figure 1 and Table 1, the absolute errors $\left|E_{r}(x)\right|$ by considering the Bernstein collocation method obtained at the collocation points $\frac{s}{n}, s=0,1, \cdots, n$ are given for $n=3$ and iterations $r=1,2,3,4$. Besides, in Figure 2, the absolute errors $\left|e_{n}(x)\right|$ of the proposed method are presented for different values $\mathrm{n}=2,3,4$ and 5-th iteration.

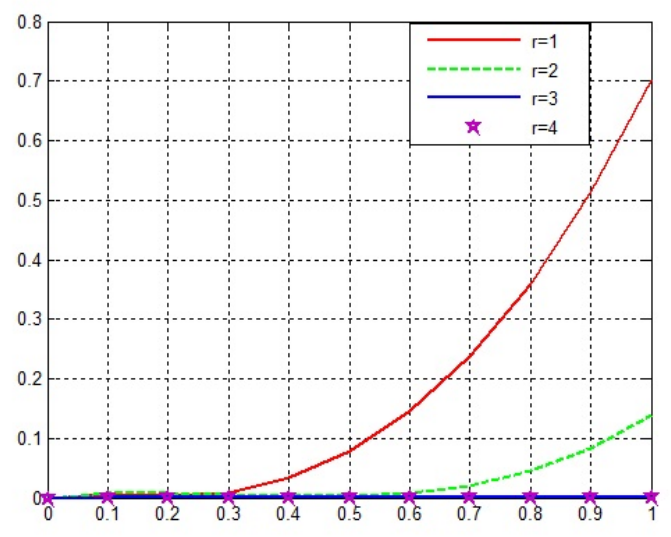

Figure 1: The numerical results of $\left|E_{r}(x)\right|$ errors for $n=3$. 
Table 1: The numerical results of $\left|E_{r}(x)\right|$ errors for $n=3$.

\begin{tabular}{|c|c|c|c|c|}
\hline $\mathrm{n}=3$ & $\mathrm{r}=1$ & $\mathrm{r}=2$ & $\mathrm{r}=3$ & $\mathrm{r}=4$ \\
\hline 0 & 0 & 0 & 0 & 0 \\
\hline 0.1 & $5.6 \times 10^{-3}$ & $7.5 \times 10^{-3}$ & $2.3 \times 10^{-4}$ & $1.7 \times 10^{-7}$ \\
\hline 0.2 & $4.3 \times 10^{-3}$ & $8.6 \times 10^{-3}$ & $2.8 \times 10^{-4}$ & $2.1 \times 10^{-7}$ \\
\hline 0.3 & $7.8 \times 10^{-3}$ & $6.3 \times 10^{-3}$ & $2.2 \times 10^{-4}$ & $1.7 \times 10^{-7}$ \\
\hline 0.4 & $3.4 \times 10^{-2}$ & $3.4 \times 10^{-3}$ & $1.3 \times 10^{-4}$ & $1.1 \times 10^{-7}$ \\
\hline 0.5 & $7.9 \times 10^{-2}$ & $2.8 \times 10^{-3}$ & $1.0 \times 10^{-4}$ & $9.3 \times 10^{-8}$ \\
\hline 0.6 & $1.5 \times 10^{-1}$ & $7.6 \times 10^{-3}$ & $2.2 \times 10^{-4}$ & $1.8 \times 10^{-7}$ \\
\hline 0.7 & $2.4 \times 10^{-1}$ & $2.1 \times 10^{-2}$ & $5.5 \times 10^{-4}$ & $4.3 \times 10^{-7}$ \\
\hline 0.8 & $3.6 \times 10^{-1}$ & $4.5 \times 10^{-2}$ & $1.2 \times 10^{-3}$ & $8.9 \times 10^{-7}$ \\
\hline 0.9 & $5.1 \times 10^{-1}$ & $8.3 \times 10^{-2}$ & $2.2 \times 10^{-3}$ & $1.6 \times 10^{-6}$ \\
\hline 1.0 & $7.0 \times 10^{-1}$ & $1.4 \times 10^{-1}$ & $3.7 \times 10^{-3}$ & $2.7 \times 10^{-6}$ \\
\hline
\end{tabular}

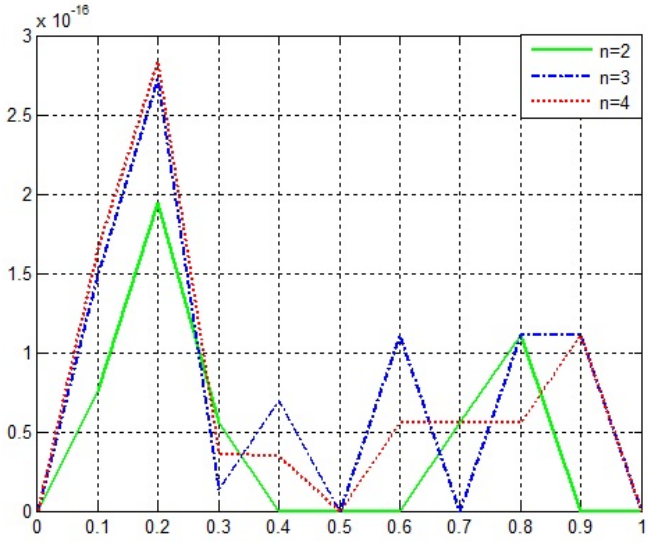

Figure 2: The numerical results of $\left|e_{n}(x)\right|$ errors for 5-th iteration.

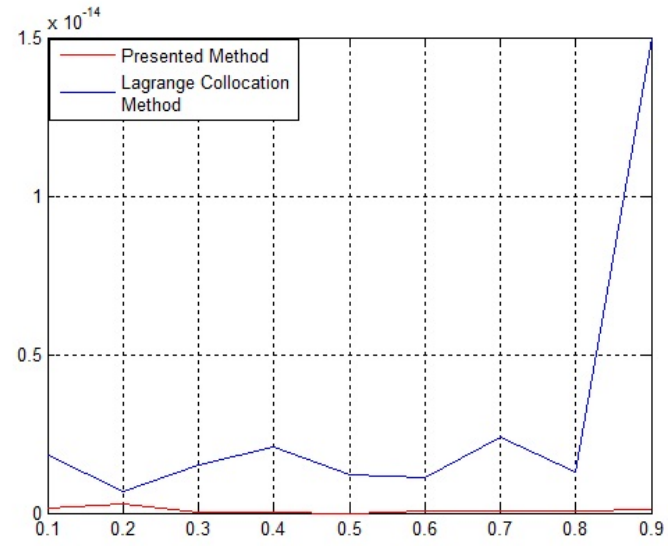

Figure 3: The comparison of numerical results of $\left|e_{n}(x)\right|$ errors for $n=4$ and 5-th iteration.

Table 2: The comparison of the $\left|e_{n}(x)\right|$ errors of Example 4.1.

\begin{tabular}{|c|c|c|c|c|c|c|c|c|}
\hline \multirow{3}{*}{$x$} & \multicolumn{9}{|c|}{ Presented Method } & \multicolumn{2}{c|}{$\begin{array}{l}\text { Lagrange Collocation } \\
\text { Method [13] }\end{array}$} \\
\cline { 2 - 9 } & \multicolumn{2}{|c|}{$\mathrm{n}=2$} & \multicolumn{2}{|c|}{$\mathrm{n}=3$} & \multicolumn{2}{c|}{$\mathrm{n}=4$} & \multicolumn{2}{c|}{$\mathrm{n}=4$} \\
\cline { 2 - 9 } & $\mathrm{r}=2$ & $\mathrm{r}=5$ & $\mathrm{r}=2$ & $\mathrm{r}=5$ & $\mathrm{r}=2$ & $\mathrm{r}=5$ & $\mathrm{r}=2$ & $\mathrm{r}=5$ \\
\hline 0.1 & $4.7 \times 10^{-5}$ & $7.6 \times 10^{-17}$ & $2.3 \times 10^{-4}$ & $1.5 \times 10^{-16}$ & $5.8 \times 10^{-5}$ & $1.7 \times 10^{-16}$ & $2.5 \times 10^{-3}$ & $1.8 \times 10^{-15}$ \\
\hline 0.2 & $4.5 \times 10^{-5}$ & $1.9 \times 10^{-16}$ & $2.8 \times 10^{-4}$ & $2.7 \times 10^{-16}$ & $3.9 \times 10^{-5}$ & $2.8 \times 10^{-16}$ & $7.9 \times 10^{-4}$ & $6.6 \times 10^{-16}$ \\
\hline 0.3 & $7.4 \times 10^{-6}$ & $5.6 \times 10^{-17}$ & $2.2 \times 10^{-4}$ & $1.4 \times 10^{-17}$ & $8.7 \times 10^{-6}$ & $3.6 \times 10^{-17}$ & $1.4 \times 10^{-3}$ & $1.5 \times 10^{-15}$ \\
\hline 0.4 & $1.1 \times 10^{-4}$ & 0 & $1.3 \times 10^{-4}$ & $6.9 \times 10^{-17}$ & $3.9 \times 10^{-6}$ & $3.5 \times 10^{-17}$ & $1.8 \times 10^{-3}$ & $2.1 \times 10^{-15}$ \\
\hline 0.5 & $2.6 \times 10^{-4}$ & 0 & $1.1 \times 10^{-4}$ & 0 & $3.5 \times 10^{-5}$ & 0 & $9.0 \times 10^{-4}$ & $1.2 \times 10^{-15}$ \\
\hline 0.6 & $4.6 \times 10^{-4}$ & 0 & $2.2 \times 10^{-4}$ & $1.1 \times 10^{-16}$ & $8.4 \times 10^{-5}$ & $5.6 \times 10^{-17}$ & $3.6 \times 10^{-3}$ & $1.1 \times 10^{-15}$ \\
\hline 0.7 & $7.1 \times 10^{-4}$ & $5.6 \times 10^{-17}$ & $5.5 \times 10^{-4}$ & 0 & $1.1 \times 10^{-4}$ & $5.6 \times 10^{-17}$ & $1.8 \times 10^{-2}$ & $2.4 \times 10^{-15}$ \\
\hline 0.8 & $1.0 \times 10^{-3}$ & $1.1 \times 10^{-16}$ & $1.2 \times 10^{-3}$ & $1.1 \times 10^{-16}$ & $3.5 \times 10^{-5}$ & $5.6 \times 10^{-17}$ & $5.5 \times 10^{-2}$ & $1.3 \times 10^{-15}$ \\
\hline 0.9 & $1.4 \times 10^{-3}$ & 0 & $2.2 \times 10^{-3}$ & $1.1 \times 10^{-16}$ & $2.3 \times 10^{-4}$ & $1.1 \times 10^{-16}$ & $1.3 \times 10^{-1}$ & $1.5 \times 10^{-14}$ \\
\hline
\end{tabular}

The absolute error results of the proposed method are compared with the results given by Maleknejad and Najafi [13] in Table 2 and Figure 3. The numerical results of the Bernstein collocation method are obtained at the collocation points $\frac{s}{n}, s=0,1, \cdots, n$ by considering the first iteration function $y_{0}(x)=2-e^{x}$. Besides, Maleknejad and Najafi have presented a collocation method based on quasilinearization technique and Lagrange basis polynomials for the first iteration function $y_{0}(x)=1-e^{x}$. According to Table 2 , the presented method has better numerical solutions than the other method for $r=2$ and $r=5$. The 
best results are also obtained for $n=2$ by the presented method, therefore, it is not necessary to enlarge $n$ since the rounding error increases while $n$ increases.

Example 4.2. Consider the following nonlinear Fredholm-Volterra integral equation:

$$
y(x)=\frac{2 x+7-7 x^{4}}{3}+\int_{-1}^{x}(x+t) y^{2}(t) d t+\int_{-1}^{1}(x-t) y(t) d t, \quad-1 \leqslant x \leqslant 1 .
$$

Exact solution of the above equation is $y(x)=2 x$. Let $y_{0}(x)=0$ be the first iteration function.

Table 3: The numerical results of $e_{\text {mean }}$ errors for Example 4.2.

\begin{tabular}{|c|c|c|c|c|c|}
\hline $\mathrm{n}$ & $\mathrm{r}=2$ & $\mathrm{r}=3$ & $\mathrm{r}=4$ & $\mathrm{r}=5$ & $\mathrm{r}=6$ \\
\hline 2 & $1.6 \times 10^{-1}$ & $3.7 \times 10^{-2}$ & $3.1 \times 10^{-3}$ & $2.6 \times 10^{-5}$ & $7.1 \times 10^{-8}$ \\
\hline 4 & $8.2 \times 10^{-2}$ & $6.4 \times 10^{-3}$ & $4.2 \times 10^{-5}$ & $1.7 \times 10^{-7}$ & $6.5 \times 10^{-8}$ \\
\hline 8 & $5.0 \times 10^{-2}$ & $1.6 \times 10^{-3}$ & $1.5 \times 10^{-6}$ & $2.6 \times 10^{-7}$ & $1.1 \times 10^{-7}$ \\
\hline 16 & $4.1 \times 10^{-2}$ & $1.1 \times 10^{-3}$ & $4.2 \times 10^{-7}$ & $3.5 \times 10^{-6}$ & $9.5 \times 10^{-7}$ \\
\hline
\end{tabular}

Mean errors of the presented method with increasing $n$ are given at the collocation points $x_{s}=-1+\frac{2 s}{n}, s=0,1, \cdots, n$ in Table 3 . We can say that the numerical results of proposed method converge more rapidly for increasing iteration $r$.

\section{Conclusions}

In this study, a collocation method based on the generalized Bernstein polynomials has been developed for the numerical solution of nonlinear Fredholm-Volterra integral equations iteratively by using the quasilinearization technique. The quadratic convergence and error bound of the Bernstein collocation method have been analyzed. Examples 4.1 and 4.2 support that the proposed method derived iteratively converges more rapidly for increasing iterations $r$. The advantage of the proposed method is not complicated and easy applicability to the nonlinear equations, because the nonlinear equations are reduced to linear equations via the quasilinearization. So the method does not need any solution methods of nonlinear equations.

\section{Acknowledgment}

This work is supported by the Scientific Research Project Coordination Unit of Pamukkale University with number 2017KRM002-193.

Some results of the present work have been reported at the International Conference on the Theory, Methods and Applications of Nonlinear Equations, held at the University of Texas A\&M, Kingsville in 2012.

\section{References}

[1] R. P. Agarwal, Y. M. Chow, Iterative methods for a fourth order boundary value problem, J. Comput. Appl. Math., 10 (1984), 203-217. 1

[2] B. Ahmad, R. Ali Khan, S. Sivasundaram, Generalized quasilinearization method for nonlinear functional differential equations, J. Appl. Math. Stochastic Anal., 16 (2003), 33-43. 1

[3] A. Akyuz Dascioglu, N. Isler, Bernstein collocation method for solving nonlinear differential equations, Math. Comput. Appl., 18 (2013), 293-300. 1

[4] A. Akyüz-Daşcioğlu, N. İşler Acar, C. Güler, Bernstein collocation method for solving nonlinear Fredholm-Volterra integrodifferential equations in the most general form, J. Appl. Math., 2014 (2014), 8 pages. 1

[5] A. C. Baird, Jr., Modified quasilinearization technique for the solution of boundary-value problems for ordinary differential equations, J. Optimization Theory Appl., 3 (1969), 227-242. 1 
[6] R. E. Bellman, R. E. Kalaba, Quasilinearization and nonlinear boundary-value problems, Modern Analytic and Computional Methods in Science and Mathematics, American Elsevier Publishing Co., Inc., New York, (1965). 1

[7] Z. Drici, F. A. McRae, J. V. Devi, Quasilinearization for functional differential equations with retardation and anticipation, Nonlinear Anal., 70 (2009), 1763-1775. 1

[8] R. T. Farouki, V. T. Rajan, Algorithms for polynomials in Bernstein form, Comput. Aided Geom. Design, 5 (1988), 1-26. 1

[9] N. İşler Acar, A. Akyüz-Daşcioğlu, A collocation method for Lane-Emden Type equations in terms of generalized Bernstein polynomials, Pioneer J. Math. Math. Sci., 12 (2014), 81-97. 1

[10] K. I. Joy, Bernstein polynomials, On-Line Geometric Modeling Notes, Visualization and Graphics Research Group, Department of Computer Science, University of California, Davis, (2000), 1-13. 1

[11] E. S. Lee, Quasilinearization and invariant imbedding, First edition, Academic Press, New York, (1968). 1

[12] G. G. Lorentz, Bernstein polynomials, Second edition, Chelsea Publishing Co., New York, (1986). 1

[13] K. Maleknejad, E. Najafi, Numerical solution of nonlinear Volterra integral equations using the idea of quasilinearization, Commun. Nonlinear Sci. Numer. Simul., 16 (2011), 93-100. 1, 2, 4.1

[14] V. B. Mandelzweig, F. Tabakin, Quasilinearization approach to nonlinear problems in physics with application to nonlinear ODEs, Comput. Phys. Comm., 141 (2001), 268-281. 1

[15] S. G. Pandit, Quadratically converging iterative schemes for nonlinear Volterra integral equations and an application, J. Appl. Math. Stochastic Anal., 10 (1997), 169-178. 1

[16] M. G. Phillips, Interpolation and approximation by polynomials, CMS Books in Mathematics/Ouvrages de Mathmatiques de la SMC, Springer-Verlag, New York, (2003). 1

[17] J. I. Ramos, Piecewise quasilinearization techniques for singular boundary-value problems, Comput. Phys. Comm., 158 (2004), 12-25. 1

[18] P.-G. Wang, Y.-H. Wu, B. Wiwatanapaphee, An extension of method of quasilinearization for integro-differential equations, Int. J. Pure Appl. Math., 54 (2009), 27-37. 1 